\title{
On link homology theories from extended cobordisms
}

\author{
Anna Beliakova and Emmanuel Wagner
}

\begin{abstract}
This paper is devoted to the study of algebraic structures leading to link homology theories. The originally used structures of Frobenius algebra and/or TQFT are modified in two directions. First, we refine 2-dimensional cobordisms by taking into account their embedding into $\mathbb{R}^{3}$. Secondly, we extend the underlying cobordism category to a 2-category, where the usual relations hold up to 2-isomorphisms. The corresponding abelian 2-functor is called an extended quantum field theory (EQFT). We show that the Khovanov homology, the nested Khovanov homology, extracted by Stroppel and Webster from Seidel-Smith construction, and the odd Khovanov homology fit into this setting. Moreover, we prove that any (strict) EQFT based on a $\mathbb{Z}_{2}$-extension of the embedded cobordism category that coincides with Khovanov homology after reducing the coefficients modulo 2 gives rise to a link invariant homology theory isomorphic to that of Khovanov.
\end{abstract}

Mathematics Subject Classification (2010). 57R58; 57M27.

Keywords. Khovanov homology, Frobenius algebra, 2-cobordism, Jones polynomial, cohomology of categories.

\section{Introduction}

In his influential paper [6], Khovanov constructed a link homology theory categorifying the Jones polynomial. During few years, this categorification was considered to be essentially unique since the underlying $(1+1)$ TQFT was known to be determined by its Frobenius system and all rank two Frobenius systems were fully classified [7]. However, in [12] Ozsvath, Rasmussen and Szabo came up with a new categorification of the Jones polynomial which agrees with Khovanov's after reducing the coefficients modulo two. The underlying algebraic structure of the odd Khovanov homology cannot be described in terms of the Frobenius algebra.

This fact attracts attention again to the question of description and classification of algebraic structures leading to link homology theories. In this paper, we provide an evidence to the fact that the appropriate algebraic structure is given by an extended quantum field theory (EQFT). A EQFT here is a 2-functor from a certain (semistrict) monoidal 2-category of cobordisms, called an extension, to an abelian category. Given 
a cobordism category by specifying its generators and relations, the 2-category is constructed by requiring the relations to be satisfied up to 2-isomorphisms. Furthermore, such a 2-category is called an extension of the original cobordism category if the automorphism group of any 1-morphism is trivial. A simple example of an extension is a $\mathbb{Z}_{2}$-extension where the 2-isomorphisms are just plus or minus the identity maps. Notice that extensions can be defined for both strict and semistrict monoidal 2-categories and the resulting EQFT will also be called strict and semistrict respectively.

The usage of the word "extension" in our setting is motivated by the fact that after replacing the original category by a group we will get a usual extension of that group. Those extensions are classified by the second cohomology classes of the group. Therefore, our approach can serve as a definition for the second cohomology of a category. A quite different notion of an extended topological field theory (ETFT) was introduced and studied in [15].

In this paper, we construct extensions of the category of 2-dimensional cobordisms $\mathrm{Cob}$ and of the category of embedded 2-cobordisms modulo the unknotting relation NesCob. In the first case, we recover the Khovanov and the odd Khovanov homologies as strict (trivial) and semistrict extensions, respectively. In the second case, we construct so-called nested Khovanov homology, extracted by Stroppel and Webster [17] from the algebraic counterpart of the Seidel-Smith construction. In addition, we show that the latter theory is equivalent to that of Khovanov. More precisely, for a given diagram $D$ let us denote by $\llbracket D \rrbracket$ its Khovanov hypercube of resolutions. Applying the Khovanov TQFT, we get a complex $F_{\mathrm{Kh}} \llbracket D \rrbracket$. On the other hand, using the nested Frobenius system defined in Section 2.3, we get the complex $F \llbracket D \rrbracket$.

Theorem 1. Given a diagram $D$ of a link $L$, the complexes $F_{\mathrm{Kh}} \llbracket D \rrbracket$ and $F \llbracket D \rrbracket$ are isomorphic.

It follows that the homotopy type of $F \llbracket D \rrbracket$ is invariant under Reidemeister moves.

Recall that in 2004 Seidel and Smith defined a link invariant homology theory using Lagrangian intersections of nilpotent slices. They conjectured that their theory is equivalent to Khovanov's after collapsing the gradings. An algebraic counterpart of the Seidel-Smith construction was given by Cautis-Kamnitzer. Finally, Stroppel and Webster made explicit computations of the Cautis-Kamnitzer differential and discovered that it behaves locally like the one in the nested Khovanov homology.

Hence, once the equivalence between the geometric construction of Seidel-Smith and the algebraic one of Cautis-Kamnitzer is established rigorously, Theorem 1 can be used to finalize the proof of the Seidel-Smith conjecture.

A similar result was independently proved by Gisa Schaefer [14].

The last result of the paper is the classification of all rank two strict $\mathbb{Z}_{2}$-extensions of NesCob.

Theorem 2. Any strict EQFT based on a $\mathbb{Z}_{2}$-extension of NesCob, which agrees with Khovanov's TQFT after reducing the coefficients modulo 2, gives rises to a link invariant homology theory isomorphic to that of Khovanov. 
A challenging open problem is to classify all semistrict EQFTs based on NesCob, which associate to a circle a rank two module. More generally, the problem is to compute the second cohomology of NesCob and construct cocycles restricting to the Schur cocycle of the symmetric group.

An interesting algebraic system underlying the categorification of the Kauffman skein module [1], [18] was proposed recently by Carter and Saito [4]. We wonder whether our approach could be extended to include their setting.

The paper is organized as follows. In the first sections we define the categories $\mathrm{Cob}, \mathrm{NesCob}$ and their extensions. Theorems 1 and 2 are proved in Section 3. In the last section, odd Khovanov homology is realized as a $\mathbb{Z}_{2}$-extension of the genus zero part of Cob.

Acknowledgment. The authors would like to thank Christian Blanchet, Aaron Lauda, Krzysztof Putyra, Alexander Shumakovitch and Catharina Stroppel for interesting discussions and to Dror Bar-Natan for the permission to use his picture of the Khovanov hypercube.

\section{The category of 2-cobordisms and its extensions}

Convention: Throughout this paper, cobordisms are maps from the left configuration of circles to the right one. The only exception is Figure 3 (taken from [2]) where the cobordism is drawn as a map from the top to the bottom.

\subsection{The category Cob}

Definition 1.1. The objects of Cob are finite ordered sets of circles. The morphisms are isotopy classes of smooth 2-dimensional cobordisms. The composition is given by gluing of cobordisms.

The category Cob is a strict symmetric monoidal category with the monoidal product given by the ordered disjoint union and the identity given by the cylinder cobordism. In particular, we obtain a natural embedding of the symmetric group in $n$ letters into the automorphism group of $n$ circles.

By using Morse theory, one can decompose any 2-cobordism into pairs of pants, caps, cups and permutations, proving the following well-known presentation of $\mathrm{Cob}$ (see e.g. [5])

Theorem 1.2. The morphisms of $\mathrm{Cob}$ are generated by

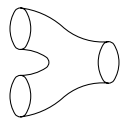

merge

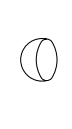

birth

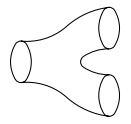

split death

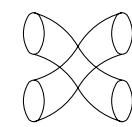

permutation 
subject to the following relations.

(1) Commutativity and co-commutativity relations:
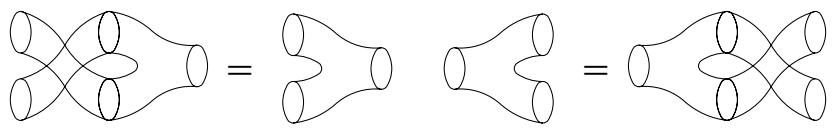

(2) Associativity and coassociativity relations:
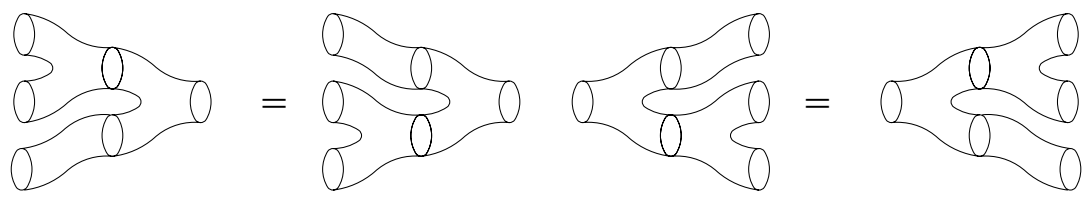

(3) Frobenius relations:

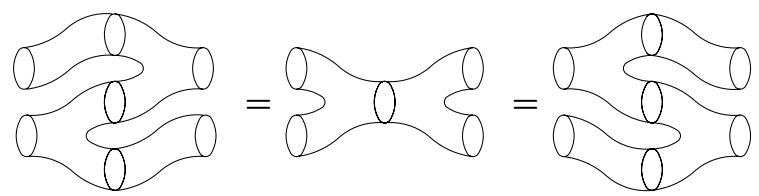

(4) Unit and counit relations:

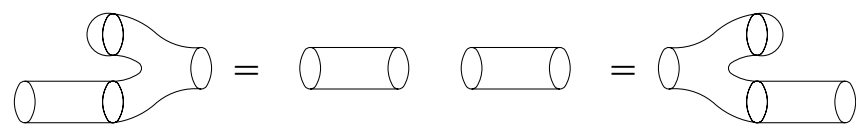

(5) Permutation relations:
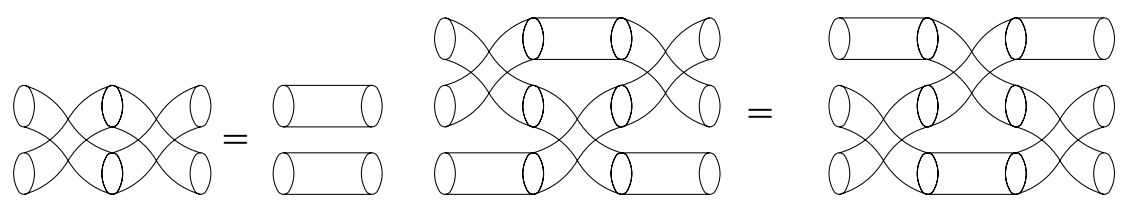

(6) Unit-permutations and counit-permutation relations:

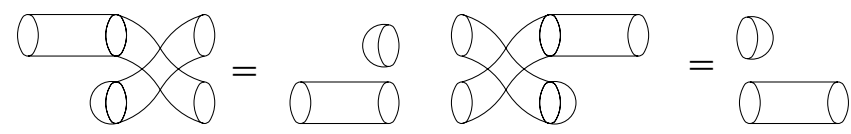

(7) Merge-permutation and split-permutation relations:
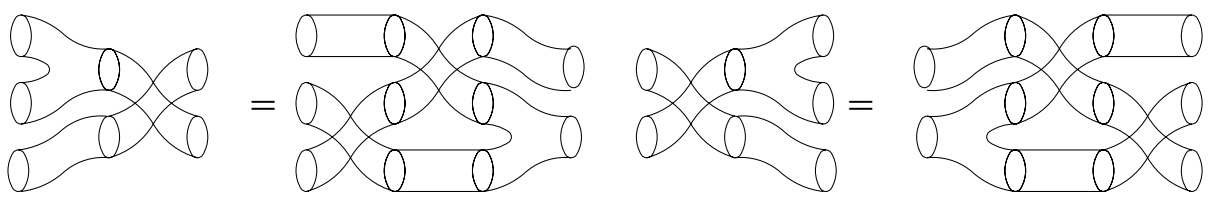

For a commutative unital ring $R$, let $R$-Mod be the category of finite projective modules over $R$. A (1+1)-dimensional topological quantum field theory (TQFT) is a symmetric (strict) monoidal functor from Cob to $R$-Mod. Such TQFTs are in $1: 1$ correspondence with so-called Frobenius systems (cf. [8]). 
One important application of Frobenius systems is Khovanov's categorification of the Jones polynomial [6].

In what follows we will assume that $\mathrm{Cob}$ is a pre-additive category. This means that we supply the set of morphisms (between any two given objects) with the structure of an abelian group by allowing formal $\mathbb{Z}$-linear combinations of cobordisms and extending the composition maps bilinearly.

1.2. Extensions of categories. Let us introduce the notion of a 2-category. A 2category is a category where any set of morphisms has a structure of a category, i.e., we allow morphisms between morphisms, called 2-morphisms. Given a 2-category $M$, the 2-morphisms of $M$ can be composed in two ways. For any three objects $a, b$, $c$ of $M$, the composition in the category $\operatorname{Mor}_{M}(a, b)$ is called vertical composition and the bifunctor $*: \operatorname{Mor}_{M}(a, b) \times \operatorname{Mor}_{M}(b, c) \rightarrow \operatorname{Mor}_{M}(a, c)$ is called horizontal composition. These compositions are required to be associative and to satisfy an interchange law (see [11] for more details).

A semistrict monoidal 2-category can be considered as a weakening of a monoidal 2-category, where monoidal and interchange rules hold up to natural isomorphisms (cf. [9], Proposition 17).

Assume that $\mathcal{C}$ is a strict monoidal category whose set of morphisms is given by generators and relations.

Definition 1.3. An extension of $\mathcal{C}$ is the semistrict monoidal 2-category Ex $\mathcal{C}$, which has the same set of objects as $\mathcal{C}$. The 1-morphisms of Ex $\mathcal{C}$ are compositions of the generating 1-morphisms of $\mathcal{C}$. The 2-morphisms are

- the identity automorphism of any 1-morphism of $\mathcal{C}$;

- a 2-isomorphism between any two 1-morphisms subject to a relation in $\mathcal{C}$.

This imposes a so-called "cocycle" condition on the set of 2-morphisms since any composition of 2-morphisms going from a given 1-morphism to itself should be equal to the identity or any closed loop of 2-morphisms is trivial.

The weak monoidal category $(M, \otimes, 1, \alpha, \lambda, \rho)$ provides an example of an extension, where $\alpha, \lambda$ and $\rho$ are considered as 2-isomorphisms and the cocycle condition holds due to MacLane's coherence theorem [11], Chapter VII. In the case when $\mathcal{C}$ is Cob-restricted to connected cobordisms (i.e., permutation is removed from the set of generators in Theorem 1.2 as well as the relations (1), (5), (6) and (7)), then any pseudo-Frobenius algebra described in [9], Proposition 25, defines an extension Ex $\mathcal{C}$. The cocycle condition holds due to Lemmas 32, 33 in [9].

Providing $C$ with the structure of a pre-additive category, we have a natural $\mathbb{Z}$ action on the set of 1 -morphisms. Restricting to $\mathbb{Z}_{2}=\{1,-1\}$, the group of two elements written multiplicatively, we can define a $\mathbb{Z}_{2}$-extension of $\mathcal{C}$ in which the 2 -morphisms are just plus or minus the identity. Note that in this case Ex $\mathcal{C}$ can be considered as a weak monoidal category, with the same set of generating 1-morphisms as $\mathcal{C}$ but with sign modified relations. 
Definition 1.4. For any cobordism category $e$, an extended quantum field theory (EQFT) based on $\ell$ is a bifunctor from Ex $C$ to $R$-Mod, mapping 2-morphisms to natural transformations of $R$-modules. The EQFT is called strict if Ex $e$ is strict.

\section{Embedded cobordisms}

Let $S^{\amalg a}$ be the disjoint union of $a$ copies of a circle smoothly embedded into a plane. Note that the embedding induces a partial order on the set of circles as follows. For two circles $c_{1}$ and $c_{2}$, we say $c_{1}<c_{2}$ if $c_{1}$ is inside $c_{2}$.

Definition 2.1. The objects of NesCob are finite collections of circles embedded into a plane. The morphisms are generated by
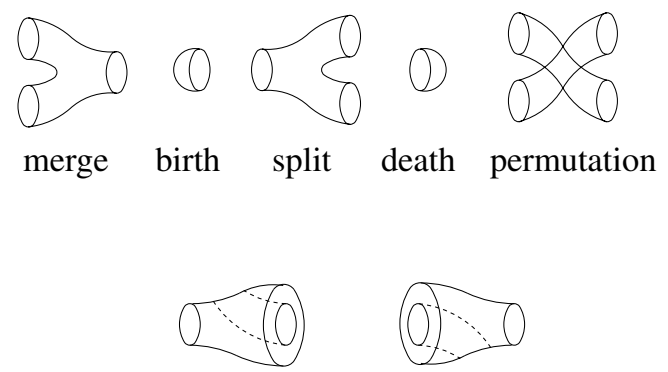

nested split nested merge

subject to the following sets of relations.

(1) Frobenius type relations:
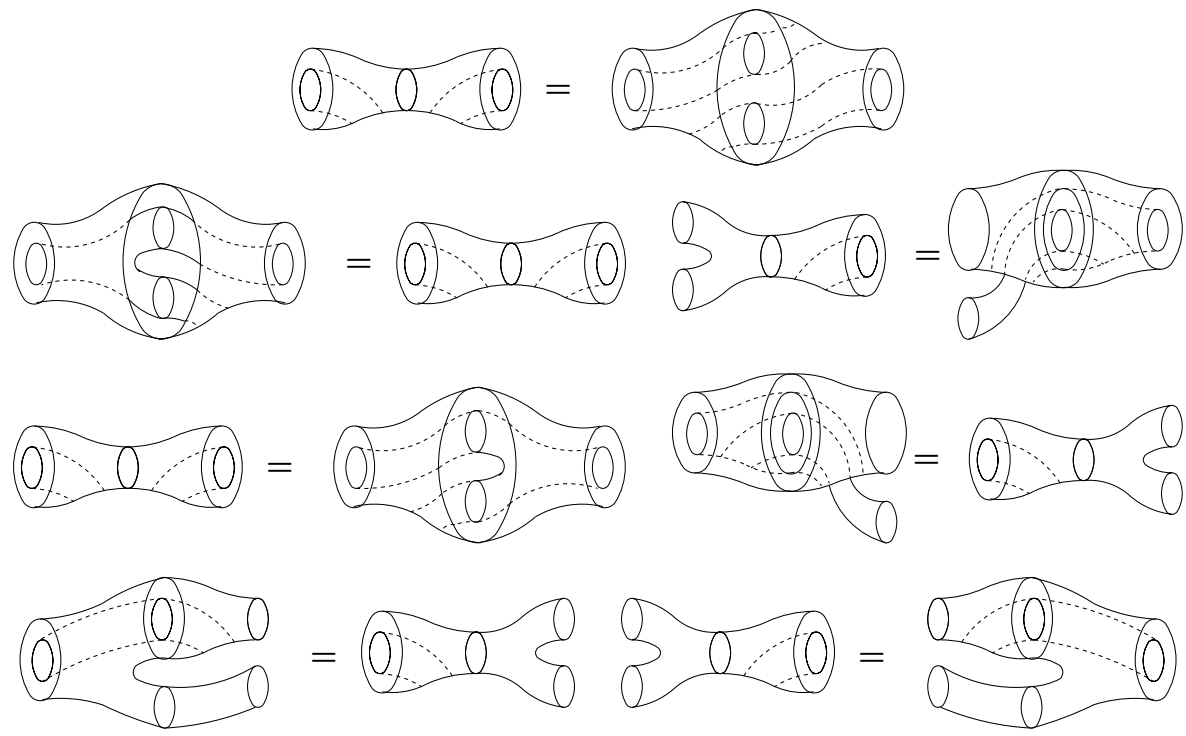
(2) Associativity type relations:

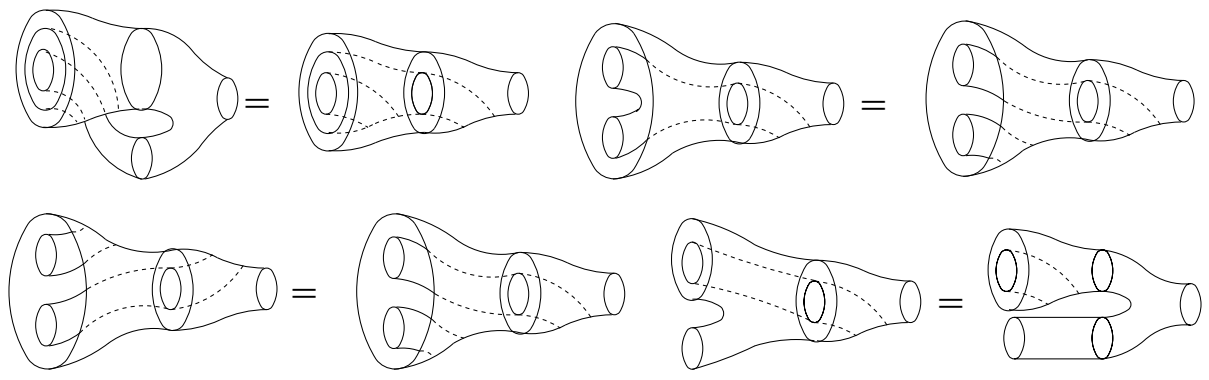

(3) Coassociativity type relations:
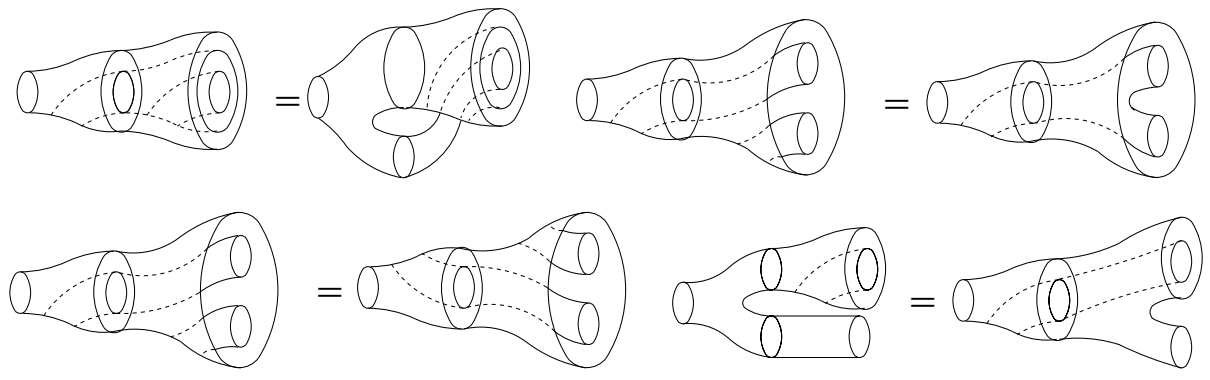

(4) Cancellation:

$$
0=0,0=0,0=0
$$

(5) Torus relation:

$$
0 \times 0=080
$$

In addition, the merge, the split, the birth, the death and the permutation are still subject to all the relations of Theorem 1.2.

Remark 2.1. The relations (1), (2), (3), and (5) of Definition 2.1 can also be described as follows:

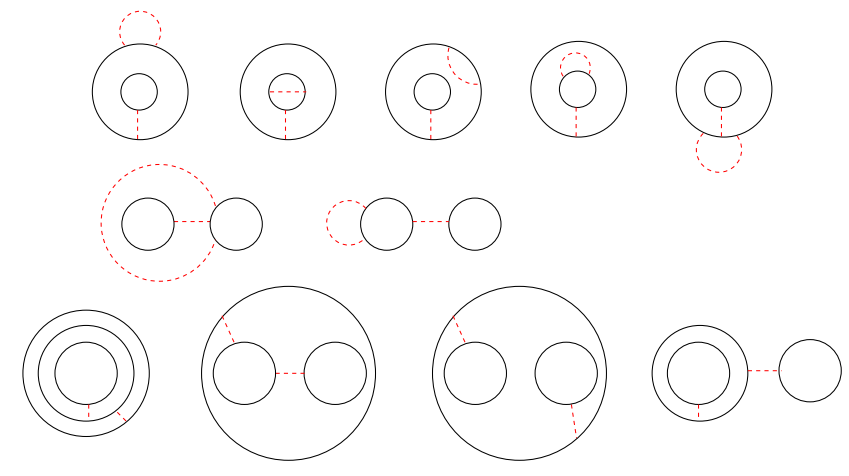




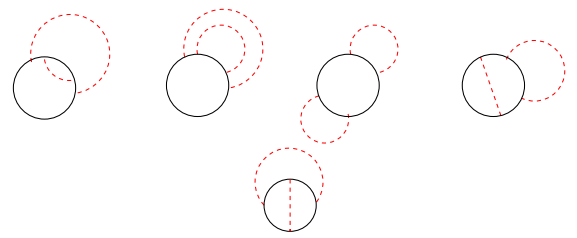

Here the black circle corresponds to the starting configuration of circles, and the dashed arcs correspond to the operations that are performed. Notice that changing the order of operations produces the two different sides of the relations in Definition 2.1.

Observe that the relations in Definition 2.1 list all possibilities to attach two chords to 1,2 or 3 circles.

The category NesCob is a symmetric strict monoidal category with a tensor product given by a partially ordered disjoint union, i.e., circles on the same level of nestedness are ordered. In particular, we obtain a natural embedding of the symmetric group into the automorphism group of any object, permuting circles not ordered by nestedness.

Any morphism in NesCob is the composite of such a permutation and the tensor product of connected morphisms of NesCob.

Lemma 2.2. Any connected morphism in NesCob has the following normal form:
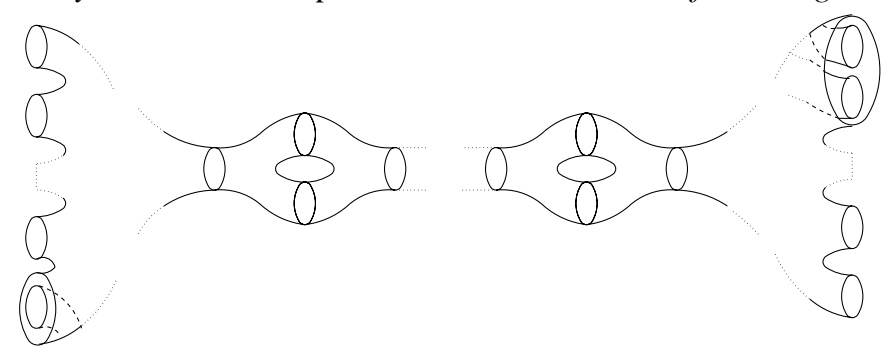

Proof. Assume that the boundary of our connected genus $g$ cobordism $C$ consists of $a$ incoming circles and $b$ outgoing ones. Let us suppose that $C$ is a composition of $B$ births, $D$ deaths, $M$ merges and $S$ splits. Then we have

$$
2-2 g-a-b=B+D-M-S, \quad a-M+B=b-S+D,
$$

or

$$
M=a+g-1+B, \quad S=b+g-1+D .
$$

We arrive at the normal form if we will be able to push all merges (resp. splits) to the incoming (resp. outgoing) boundary of $C$. From the above formulas we see that $B$ merges and $D$ splits will cancel with the births and deaths, respectively, and $g$ splits and merges put together will create $g$ handles. The remaining $a-1$ merges commute with any split (nested or not nested) due to the Frobenius type relations. Finally using the associativity type relations, we can commute nested and unnested merges (resp. splits) and arrive at the form in Figure 1.

Furthermore applying the torus relation, we are left with the normal form. 


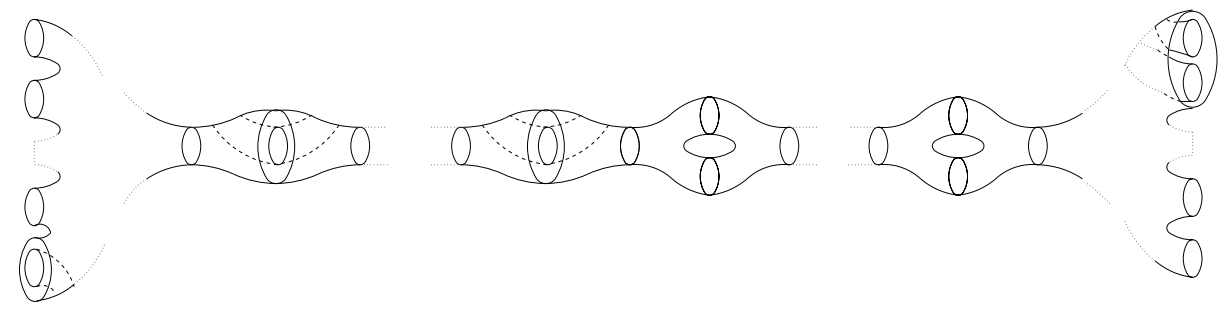

Figure 1. Normal form with inner and outer handles.

\subsection{Embedded cobordisms}

Definition 2.3. A smoothly embedded 2-dimensional cobordism from $S^{\amalg a}$ to $S^{\amalg b}$ is a pair $(\Sigma, \phi)$, where $\Sigma$ is a smooth 2-dimensional surface whose boundary consists of $a+b$ circles and $\phi: \Sigma \hookrightarrow \mathbb{R}^{2} \times[0,1]$ is a smooth embedding such that $\left.\phi\right|_{\partial \Sigma} \cap$ $\mathbb{R}^{2} \times\{0\}=S^{\amalg a}$ and $\left.\phi\right|_{\partial \Sigma} \cap \mathbb{R}^{2} \times\{1\}=S^{\amalg b}$.

Definition 2.4. The objects of EmbCob are circles smoothly embedded into a plane. The morphisms are isotopy classes of smoothly embedded 2-dimensional cobordisms subject to the unknotting relation:

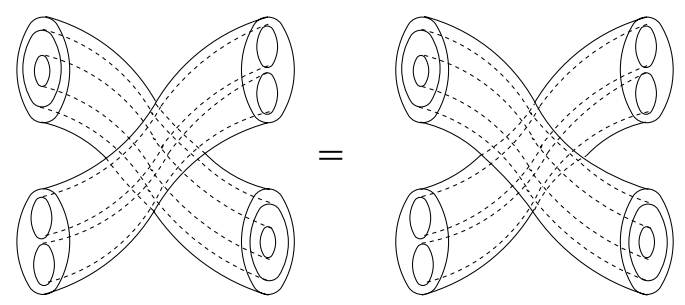

The composition is given by gluing along the boundary.

The category EmbCob is again a symmetric strict monoidal category with a tensor product given by the partially ordered disjoint union and with the action of the permutation group depending on nestedness.

Theorem 2.5. The category EmbCob is isomorphic to the category NesCob.

Proof. By [5], any smooth 2-cobordism allows a pair of pants decomposition. Modulo the unknotting relation, there are two ways to embed a pair of pants into $\mathbb{R}^{3}$, providing the list of generators in Definition 2.1. The relations do not change the isotopy class of an embedded cobordism and allow to bring it into a normal form. It remains just to say that the normal forms of two equivalent connected cobordisms coincide. 
2.2. Strict $\mathbb{Z}_{\mathbf{2}}$-extension. Assume that NesCob is a pre-additive category.

Definition 2.6. Let $\mathrm{NesCob}_{1}$ be the strict monoidal 2-category obtained from NesCob by replacing the torus relation with

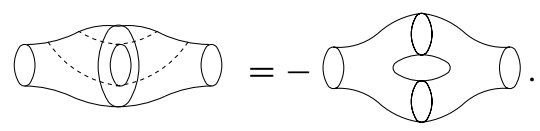

Lemma 2.7. $\mathrm{NesCob}_{1}$ is a $\mathbb{Z}_{2}$-extension of $\mathrm{NesCob}$.

Proof. It remains to show that the automorphism group of any 1-morphism is trivial. The only non-trivial 2-morphism corresponds to the torus relation T1. Both sides of T1 are compositions of two generating cobordisms. Imaging the generating cobordisms as edges, the T1-relation can be viewed as a rectangular face. It suffices to check that any cube with a T1-face has an even number of anti-commutative faces. This is a simple case by case check. Like in Remark 2.1, all such cubes appear by changing the order of attaching 3 chords to one or two circles. The resulting 10 cubes are depicted in Figure 2. In addition one can also easily check that the torus relation behaves well with respect to the permutation.

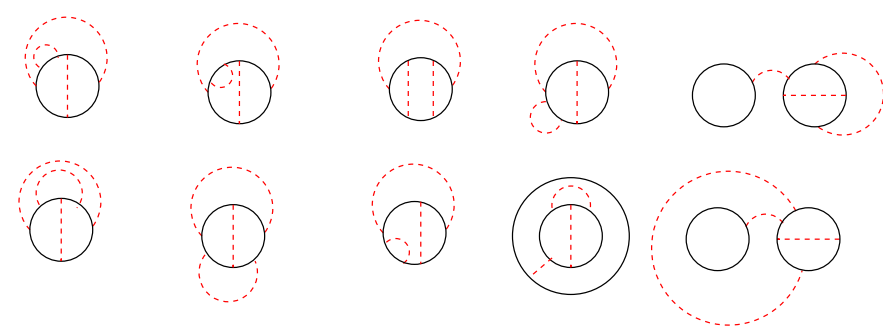

Figure 2. Cocycle conditions for $\mathrm{NesCob}_{1}$.

Remark 2.2. Notice that any element of $\mathrm{NeSCob}_{1}$ does still have a normal form which corresponds to the usual one plus the information on the parity of the number of inner 1-handles in Figure 1.

2.3. Nested Frobenius system. In this section we construct a strict EQFT based on $\mathrm{NesCob}_{1}$, as proposed by Stroppel [16].

As in [6], let us consider the 2-dimensional module $A:=\mathbb{Z}[t]\langle\mathbb{1}, X\rangle$ over the polynomial ring $R:=\mathbb{Z}[t]$ in one variable. We denote by $\mathbb{1}$ the image of 1 under the embedding $\eta: R \rightarrow A$. For $\varepsilon \in\{0,1\}$, we define two kinds of multiplication 
$m_{\varepsilon}: A \otimes A \rightarrow A$ as follows:

$$
m_{\varepsilon}:\left\{\begin{array}{l}
\mathbb{1} \otimes \mathbb{1} \mapsto \mathbb{1}, \\
\mathbb{1} \otimes X \mapsto X, \\
X \otimes \mathbb{1} \mapsto(-1)^{\varepsilon} X, \\
X \otimes X \mapsto(-1)^{\varepsilon} t .
\end{array}\right.
$$

Further, we define two comultiplications $\Delta_{\varepsilon}: A \rightarrow A \otimes A$ and a counit $\epsilon: A \rightarrow R$ as follows.

$$
\Delta_{\varepsilon}:\left\{\begin{array} { l } 
{ \mathbb { 1 } \mapsto X \otimes \mathbb { 1 } + ( - 1 ) ^ { \varepsilon } \mathbb { 1 } \otimes X , } \\
{ X \mapsto X \otimes X + ( - 1 ) ^ { \varepsilon } t \mathbb { 1 } \otimes \mathbb { 1 } , }
\end{array} \quad \quad \quad \left\{\begin{array}{l}
\mathbb{1} \mapsto 0, \\
X \mapsto 1 .
\end{array}\right.\right.
$$

The functor $F:$ NesCob $_{1} \rightarrow R$-Mod maps any object $S^{\amalg a}$ to $A^{\otimes a}$ and is defined on the generating morphisms as follows:

$$
\begin{aligned}
F\left(\sum 0\right) & =m_{0}, & F(\sigma \delta) & =\Delta_{0}, \\
F(\theta 0) & =m_{1}, & F(\sigma \theta) & =\Delta_{1}, \\
F(\theta) & =\eta, & F(\theta) & =\epsilon,
\end{aligned}
$$

Whenever a cobordism involves nested circles the convention is that in $A \otimes A$ the first factor corresponds to the inner circle. It is easy to see that $F$ preserves all the relations listed in Definition 2.1.

Let us introduce a grading on $A$ by putting

$$
\operatorname{deg}(t):=-4, \quad \operatorname{deg}(X):=-1, \quad \operatorname{deg}(\mathbb{1}):=1 .
$$

On the tensor product $A^{\otimes n}$ the grading is given by $\operatorname{deg}\left(a_{1} \otimes \cdots \otimes a_{n}\right):=\operatorname{deg}\left(a_{1}\right)+$ $\cdots+\operatorname{deg}\left(a_{n}\right)$.

There exist a natural grading on NesCob given by the Euler characteristic of cobordisms. As in Khovanov's case if $t=0, F$ is grading preserving.

\section{Nested Khovanov homology}

3.1. Khovanov's hypercube. Suppose that we have a generic diagram $D$ of an oriented link $L$ in $S^{3}$ with $c$ crossings. By resolving crossings of $D$ in two ways as prescribed by the Kauffman skein relations, one can associate to $D$ a $c$-dimensional cube of resolutions (compare [6] or [2]). The vertices of the cube correspond to the configurations of circles obtained after smoothing of all crossings in $D$. For any crossing, two different smoothings are allowed: the 0 - and the 1-smoothing. Therefore, we have $2^{c}$ vertices. After numbering the crossings of $D$, we can label 


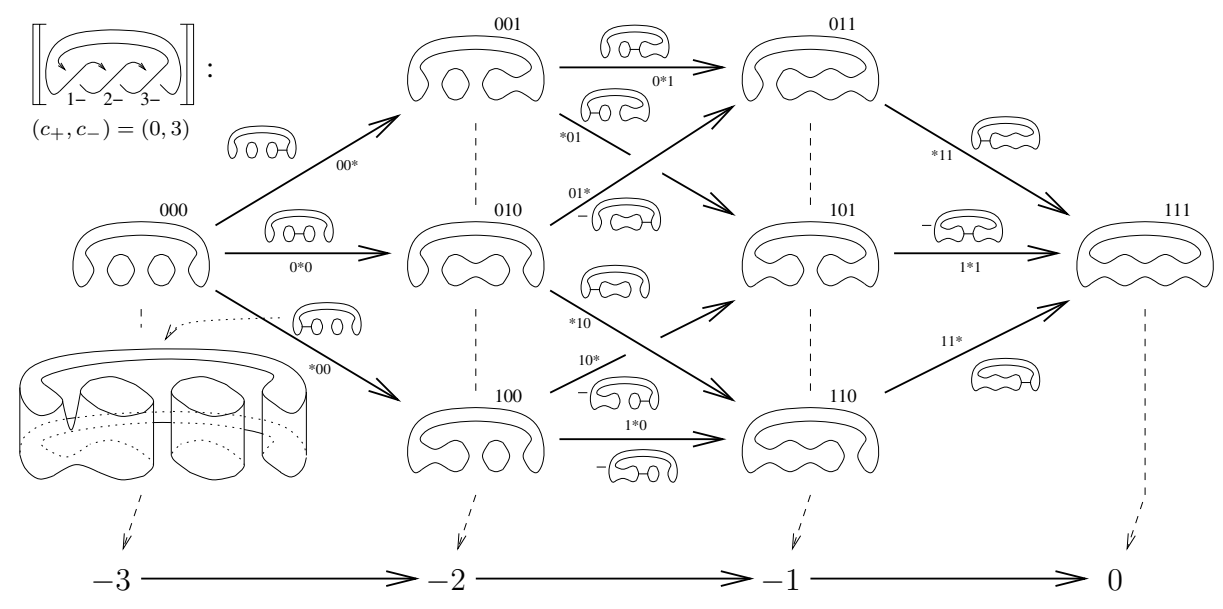

Figure 3. The cube of resolutions for the trefoil.

the vertices of the cube by $c$-letter strings of 0 's and 1's, specifying the smoothing chosen at each crossing. The cube is skewered along its main diagonal, from $00 \ldots 0$ to $11 \ldots 1$. The number 1 in the labeling of a vertex is equal to its 'height' $k$. The cube is displayed in such a way that the vertices of height $k$ project down to the point $r:=k-c_{-}$, where $c_{ \pm}$are the numbers of positive resp. negative crossings in $D$ (see Figure 3).

Two vertices of the hypercube are connected by an edge if their labellings differ by one letter. In Figure 3, this letter is labeled by $*$. The edges are directed (from the vertex where this letter is 0 to the vertex where it is 1). The edges correspond to a saddle cobordisms from the tail configuration of circles to the head configuration (compare Figure 3).

We denote this hypercube of resolutions by $\llbracket D \rrbracket$, and would like to interpret it as a complex. The $r$-th chain "space" $\llbracket D \rrbracket^{r}$ is a formal direct sum of the $\frac{c !}{k !(c-k) !}$ "spaces" at height $k$ in the hypercube and the sum of "maps" with tails at height $k$ defines the $r$-th differential. To achieve $\partial^{2}=0$, we assign a minus to any edge which has an odd number of 1's before $*$ in its labeling.

Applying (1+1) TQFT $F_{\mathrm{Kh}}$ to $\llbracket D \rrbracket$, which sends any merge to $m_{0}$ and any split to $\Delta_{0}$, we get a complex of $R$-modules $\left(F_{\mathrm{Kh}} \llbracket D \rrbracket, \partial_{\mathrm{Kh}}\right)$. Its graded homology groups, known as Khovanov homology, are link invariants and the graded Euler characteristic is given by the Jones polynomial.

3.2. Nested homology. Applying $F$ to the Khovanov hypercube, one can define a chain complex as follows. The $r$-th chain group will be $F \llbracket D \rrbracket^{r}$, the image of $\llbracket D \rrbracket^{r}$ after applying the functor $F$, and the maps are defined by applying $F$ to the corresponding cobordisms. The main difference to the Khovanov case is that here not all faces are commutative. More precisely, the square corresponding to the torus 
relation (T1) is anti-commutative. However, by the definition of the $\mathbb{Z}_{2}$-extension, the 2-cochain $\psi \in H^{2}(B, \mathbb{Z} / 2 \mathbb{Z})$ ( $B$ is the hypercube) which associates 1 to any anticommutative face of the hypercube and -1 to any commutative one is a cocycle, i.e., it vanishes on the boundary of any cube. Since the cube is contractible, any cocycle is a coboundary. Consequently, there exists a function $\epsilon: E \rightarrow \mathbb{Z}_{2}$ from the set of edges of the hypercube to $\mathbb{Z}_{2}$, called a sign assignment, such that $\epsilon\left(e_{1}\right) \epsilon\left(e_{2}\right) \epsilon\left(e_{3}\right) \epsilon\left(e_{4}\right)=$ $\psi(D)$ for any four edges $e_{1}, \ldots, e_{4}$ forming a square $D$. Hence, multiplying edges of the hypercube by the signs $\epsilon$, we get a chain complex $\left(F \llbracket D \rrbracket, \partial_{\epsilon}\right)$. It is easy to see that this complex is independent on the choice of a sign assignment.

Lemma 3.1. Given two sign assignments $\epsilon$ and $\epsilon^{\prime}$, the chain complexes $\left(F \llbracket D \rrbracket, \partial_{\epsilon}\right)$ $\left(F \llbracket D \rrbracket, \partial_{\epsilon^{\prime}}\right)$ are isomorphic.

Proof. The product $\epsilon \epsilon^{\prime}$ is a 1-cocycle. Since the hypercube is contractible, this 1cocycle is a coboundary of a 0 -cochain $\eta: V \rightarrow \mathbb{Z}_{2}$, where $V$ is the set of vertices of the hypercube. The identity map times $\eta$ provides the required isomorphism.

In the case when $t=0$ the homology groups of $(F \llbracket D \rrbracket, \partial)$ are graded and the graded Euler characteristic coincides with the Jones polynomial. If $t \neq 0$, then deg defines a filtration on our chain complex, similar to the one considered by Lee [10].

Our next aim is to show that the complex we just constructed is isomorphic to the Khovanov complex.

3.3. Proof of Theorem 1. We have to show that $(F \llbracket D \rrbracket, \partial)$ and $\left(F_{\mathrm{Kh}} \llbracket D \rrbracket, \partial_{\mathrm{Kh}}\right)$ are isomorphic.

For any circle $c$ in $S^{\amalg k}$, we define nes $(c)$ to be the number of circles in $S^{\amalg k}$ containing $c$ inside. Further, we define an endomorphism ${ }^{1} \phi_{k}$ of $F\left(S^{\amalg k}\right)$ as follows: For a copy of $A$ associated with $c$, we put

$$
\phi_{c}:\left\{\begin{array}{l}
\mathbb{1}, \mathbb{1}, \\
X \mapsto(-1)^{\operatorname{nes}(c)} X .
\end{array}\right.
$$

Then $\phi_{k}$ is the composition of $\phi_{c}$ for all circles in $S^{\amalg k}$. By abuse of notation $\phi_{k}$ depends not only on $k$ but also on the configuration of circles in $S^{\amalg k}$.

Given a link diagram $D$ with $d$ crossings, consider two Khovanov's hypercubes of resolutions associated with $D$. Apply $F$ to one of them and $F_{\mathrm{Kh}}$ to the other, and do not use any sign assignment, i.e., all squares in $F_{\mathrm{Kh}} \llbracket D \rrbracket$ are commutative. Further, observe that with each vertex of the hypercube there is associated a copy of $A^{\otimes k}$ for a certain $k$. Applying $\phi_{k}$ to any such vertex, we get a map $\Phi$ with the source ( $\left.F \llbracket D \rrbracket, \partial\right)$ and the target $\left(F_{\mathrm{Kh}} \llbracket D \rrbracket, \partial_{\mathrm{Kh}}\right)$, without any sign assignments. Our next goal is to see that there exists a sign assignment on the $(d+1)$-dimensional hypercube $\llbracket D \rrbracket \times[0,1]$ making $\Phi$ to a chain map.

\footnotetext{
${ }^{1}$ This map was suggested to us by Alexander Shumakovitch.
} 
For this, it is enough to check that each 3-dimensional cube in this $(d+1)$ dimensional hypercube contains an even number of anti-commutative faces. Note that there are three different cases: (1) the cube is contained in the source hypercube, (2) the cube is contained in the target hypercube, (3) the cube contains exactly one face in the source hypercube and one face in the target hypercube. The first case follows from Lemma 2.7 and the second from the fact that $F_{\mathrm{Kh}}$ is a $(1+1)$ TQFT. The third case relies on a case by case check. Note that all faces in the source hypercube correspond to relations in $\mathrm{NesCob}_{1}$. Hence, we have to check the claim for any cube, whose upper face is a relation in $\mathrm{NesCob}_{1}$, the lower face is the corresponding Khovanov square and whose vertical edges are labeled by $\Phi$. In addition, since the map $\phi_{c}$ depends on nes $(c)$ explicitly, we have to ensure that the claim holds after changing the nestedness of each circle by one. The tables below show that any cube of type (3) does have only commutative or anti-commutative faces. It is left to the reader to check that all cubes of type (3) do have an even number of anti-commutative faces. For this one has to consider all cubes where the upper face corresponds to one of the relations in Definition 2.1. Moreover, each cube should be checked twice for different nestedness modulo 2 .

$\bigcirc$\begin{tabular}{|c|c|c|c|c|}
\hline & $m_{1}$ & $\phi \circ m_{1}$ & $\phi$ & $m_{0} \circ \phi$ \\
\hline $\mathbb{1} \otimes \mathbb{1}$ & $\mathbb{1}$ & $\mathbb{1}$ & $\mathbb{1} \otimes \mathbb{1}$ & $\mathbb{1}$ \\
\hline $\mathbb{1} \otimes X$ & $X$ & $X$ & $\mathbb{1} \otimes X$ & $X$ \\
\hline$X \otimes \mathbb{1}$ & $-X$ & $-X$ & $-X \otimes \mathbb{1}$ & $-X$ \\
\hline$X \otimes X$ & $-t$ & $-t$ & $-X \otimes X$ & $-t$ \\
\hline
\end{tabular}

$\bigcirc$\begin{tabular}{|c|c|c|c|c|}
\hline & $m_{1}$ & $\phi \circ m_{1}$ & $\phi$ & $m_{0} \circ \phi$ \\
\hline $\mathbb{1} \otimes \mathbb{1}$ & $\mathbb{1}$ & $\mathbb{1}$ & $\mathbb{1} \otimes \mathbb{1}$ & $\mathbb{1}$ \\
\hline $\mathbb{1} \otimes X$ & $X$ & $-X$ & $-\mathbb{1} \otimes X$ & $-X$ \\
\hline$X \otimes \mathbb{1}$ & $-X$ & $X$ & $X \otimes \mathbb{1}$ & $X$ \\
\hline$X \otimes X$ & $-t$ & $-t$ & $-X \otimes X$ & $-t$ \\
\hline
\end{tabular}

\begin{tabular}{|c|c|c|c|c|}
\hline & $\Delta_{1}$ & $\phi \circ \Delta_{1}$ & $\phi$ & $\Delta_{0} \circ \phi$ \\
\hline $\mathbb{1}$ & $X \otimes \mathbb{1}-\mathbb{1} \otimes X$ & $-X \otimes \mathbb{1}-\mathbb{1} \otimes X$ & $\mathbb{1}$ & $X \otimes \mathbb{1}+\mathbb{1} \otimes X$ \\
\hline$X$ & $X \otimes X-t \mathbb{1} \otimes \mathbb{1}$ & $-X \otimes X-t \mathbb{1} \otimes \mathbb{1}$ & $X$ & $X \otimes X+t \mathbb{1} \otimes \mathbb{1}$ \\
\hline
\end{tabular}

\begin{tabular}{|c|c|c|c|c|}
\hline & $\Delta_{1}$ & $\phi \circ \Delta_{1}$ & $\phi$ & $\Delta_{0} \circ \phi$ \\
\hline $\mathbb{1}$ & $X \otimes \mathbb{1}-\mathbb{1} \otimes X$ & $X \otimes \mathbb{1}+\mathbb{1} \otimes X$ & $\mathbb{1}$ & $X \otimes \mathbb{1}+\mathbb{1} \otimes X$ \\
\hline$X$ & $X \otimes X-t \mathbb{1} \otimes \mathbb{1}$ & $-X \otimes X-t \mathbb{1} \otimes \mathbb{1}$ & $-X$ & $-X \otimes X-t \mathbb{1} \otimes \mathbb{1}$ \\
\hline
\end{tabular}

$\bigcirc \bigcirc$\begin{tabular}{|c|c|c|c|c|}
\hline & $m_{0}$ & $\phi \circ m_{0}$ & $\phi$ & $m_{0} \circ \phi$ \\
\hline $\mathbb{1} \otimes \mathbb{1}$ & $\mathbb{1}$ & $\mathbb{1}$ & $\mathbb{1} \otimes \mathbb{1}$ & $\mathbb{1}$ \\
\hline $\mathbb{1} \otimes X$ & $X$ & $X$ & $\mathbb{1} \otimes X$ & $X$ \\
\hline$X \otimes \mathbb{1}$ & $X$ & $X$ & $X \otimes \mathbb{1}$ & $X$ \\
\hline$X \otimes X$ & $t$ & $t$ & $X \otimes X$ & $t$ \\
\hline
\end{tabular}




$\bigcirc \quad$\begin{tabular}{|c|c|c|c|c|}
\hline & $m_{0}$ & $\phi \circ m_{0}$ & $\phi$ & $m_{0} \circ \phi$ \\
\hline $\mathbb{1} \otimes \mathbb{1}$ & $\mathbb{1}$ & $\mathbb{1}$ & $\mathbb{1} \otimes \mathbb{1}$ & $\mathbb{1}$ \\
\hline $\mathbb{1} \otimes X$ & $X$ & $-X$ & $-\mathbb{1} \otimes X$ & $-X$ \\
\hline$X \otimes \mathbb{1}$ & $X$ & $-X$ & $-X \otimes \mathbb{1}$ & $-X$ \\
\hline$X \otimes X$ & $+t$ & $+t$ & $+X \otimes X$ & $+t$ \\
\hline
\end{tabular}

$\odot$\begin{tabular}{c|c|c|c|c|}
\hline & $\Delta_{0}$ & $\phi \circ \Delta_{0}$ & $\phi$ & $\Delta_{0} \circ \phi$ \\
\hline $\mathbb{1}$ & $X \otimes \mathbb{1}+\mathbb{1} \otimes X$ & $X \otimes \mathbb{1}+\mathbb{1} \otimes X$ & $\mathbb{1}$ & $X \otimes \mathbb{1}+\mathbb{1} \otimes X$ \\
\hline$X$ & $X \otimes X+t \mathbb{1} \otimes \mathbb{1}$ & $X \otimes X+t \mathbb{1} \otimes \mathbb{1}$ & $X$ & $X \otimes X+t \mathbb{1} \otimes \mathbb{1}$ \\
\hline
\end{tabular}

\begin{tabular}{|c|c|c|c|c|}
\hline & $\Delta_{0}$ & $\phi \circ \Delta_{0}$ & $\phi$ & $\Delta_{0} \circ \phi$ \\
\hline $\mathbb{1}$ & $X \otimes \mathbb{1}+\mathbb{1} \otimes X$ & $-X \otimes \mathbb{1}-\mathbb{1} \otimes X$ & $\mathbb{1}$ & $X \otimes \mathbb{1}+\mathbb{1} \otimes X$ \\
\hline$X$ & $X \otimes X+t \mathbb{1} \otimes \mathbb{1}$ & $X \otimes X+t \mathbb{1} \otimes \mathbb{1}$ & $-X$ & $-X \otimes X-t \mathbb{1} \otimes \mathbb{1}$ \\
\hline
\end{tabular}

To finish, observe that the map $\Phi$ composed with a sign assignment is clearly invertible, and hence is the desired isomorphism.

3.4. Proof of Theorem 2. Let us search for further strict $\mathbb{Z}_{2}$-extensions of NesCob systematically. For $e_{i} \in \mathbb{Z}_{2}$ with $i=1, \ldots, 10$ we put

$$
\begin{gathered}
m_{0}:\left\{\begin{array}{l}
\mathbb{1} \otimes \mathbb{1} \mapsto e_{1} \mathbb{1}, \\
\mathbb{1} \otimes X \mapsto e_{2} X, \\
X \otimes \mathbb{1} \mapsto e_{2} X, \\
X \otimes X \mapsto 0,
\end{array} \quad m_{1}:\left\{\begin{array}{l}
\mathbb{1} \otimes \mathbb{1} \mapsto e_{5} \mathbb{1}, \\
X \otimes \mathbb{1} \mapsto e_{7} X, \\
X \otimes X \mapsto 0,
\end{array}\right.\right. \\
\Delta_{0}:\left\{\begin{array}{l}
\mathbb{1} \mapsto e_{3}(X \otimes \mathbb{1}+\mathbb{1} \otimes X), \\
X \mapsto e_{4} X \otimes X,
\end{array} \quad \Delta_{1}:\left\{\begin{array}{l}
\mathbb{1} \mapsto e_{8} X \otimes \mathbb{1}+e_{9} \mathbb{1} \otimes X, \\
X \mapsto e_{10} X \otimes X .
\end{array}\right.\right.
\end{gathered}
$$

The relations in Definition 2.1 should hold up to sign for any EQFT. They impose the following relations on $e_{i}$ :

$e_{1}=e_{2}$ and $e_{3}=e_{4}$ (follows from the ordinary Frobenius relation);

$e_{7} e_{8}=e_{5} e_{9}$ (follows from Frobenius type relation (1), 3. row, in Definition 2.1);

$e_{6}=e_{5}$ and $e_{9}=e_{1} 0$ (follows from Frobenius type relation (1), 4. row, in Definition 2.1).

Modulo these identities, there are 5 free parameters, i.e., 32 cases to consider. It is a simple check that all of them produce the Khovanov or nested Khovanov Frobenius system after changing the sign of one or two operations.

It remains to construct an isomorphism between, say, nested Khovanov complex and the one where $m_{0}$ is replaced by $-m_{0}$. Let us consider the map between two nested Khovanov hypercubes which is identity on all vertices, except of the tails of edges corresponding to $m_{0}$ where the map is minus the identity. As in the proof of Theorem 1, the cone of this map is a hypercube of a dimension one bigger. Let us convince ourselves that all 3-dimensional cubes of that hypercube have an even number of 
anti-commutative faces. We have to check only cubes whose upper horizontal faces belong to the nested Khovanov complex, the bottom horizontal face to the nested Khovanov with $m_{0}$ replaced by $-m_{0}$, and whose vertical edges are given by our map. If the upper horizontal face has an even number of $m_{0}$ maps, then the cube has an even number of vertical anti-commutative faces. If it has an odd number of $m_{0}$ maps, then there is an odd number of vertical anti-commutative faces, but either the top or the bottom face is anti-commutative. Hence, like in the proof of Theorem 1, there exists a sign arrangement on this hypercube providing the desired isomorphism.

\section{Odd Khovanov homology}

Let us show that the Odd Khovanov homology introduced in [12] fits in our setting.

\subsection{The extension OddCob}

Definition 4.1. The category OddCob is defined as follows: The objects of OddCob are finite ordered sets of circles. The morphisms are generated by

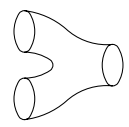

merge birth

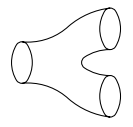

split
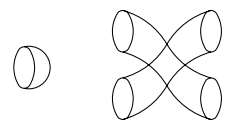

death permutation

subject to the following sets of relations.

(1) Commutativity and co-commutativity relation:
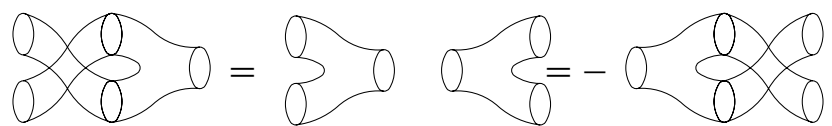

(2) Associativity and coassociativity relations
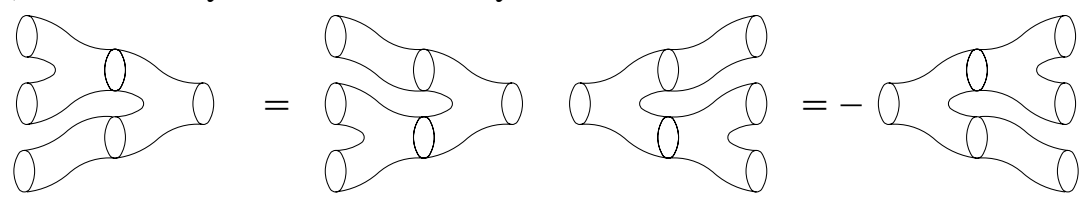

(3) Frobenius relations:

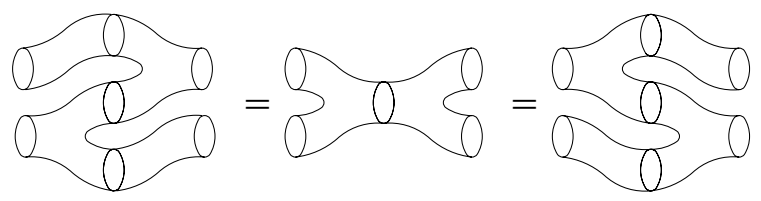

(4) Unit and counit relations:

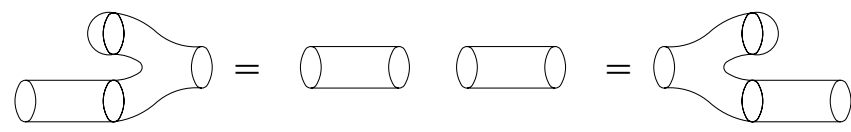


(5) Permutation relations:
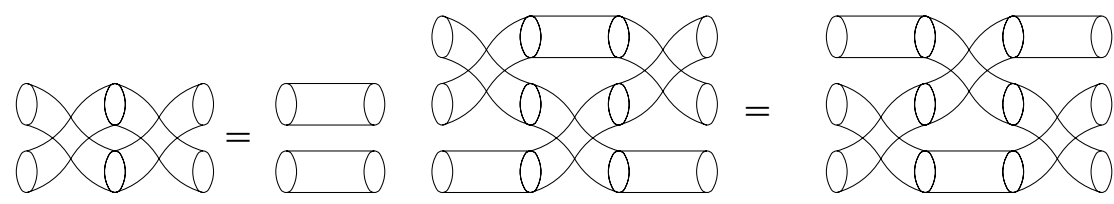

(6) Unit-permutations and counit-permutation relations:

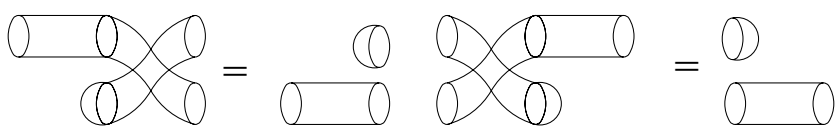

(7) Merge-permutation and split-permutation relations:
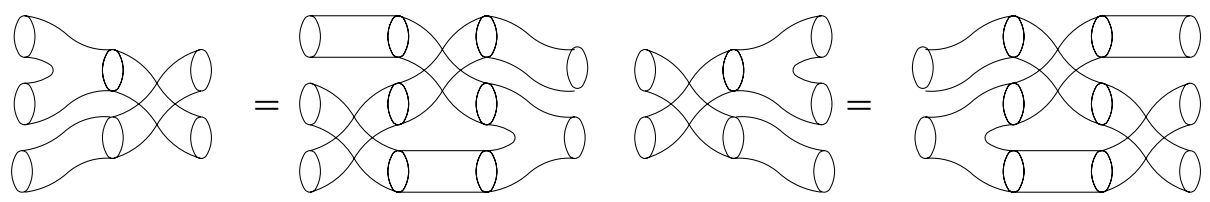

(8) Commutation relations:
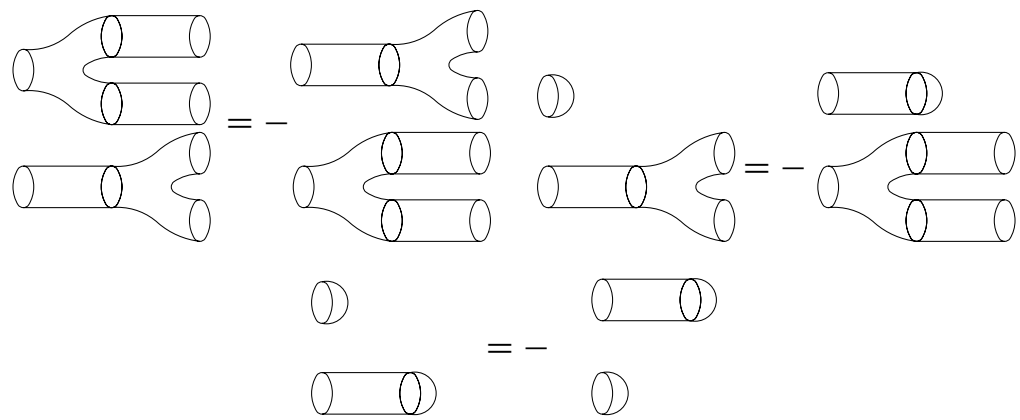

(9) Torus and sphere annihilation:

$$
Q=0 \Omega 80=0
$$

All the other commutation relations hold with plus sign.

Note. Observe that not all relations in Definition 4.1 are independent. The torus annihilation relation can be deduced from the others by first twisting the two handles of the punctured torus by means of the first permutation relation in (5), and then applying (1) to both ends.

Remark 4.1. Viewing the relations between cobordisms as 2-morphisms, one can check that OddCob is a semistrict monoidal 2-category. The monoidal structure $\nabla$ on 
OddCob can then be defined as follows. Let us endow the 1-morphisms in OddCob with the following $\mathbb{Z} / 2 \mathbb{Z}$-grading:

$$
\begin{aligned}
\operatorname{sd}(\xi 0) & =0, \quad \operatorname{sd}(\sigma \delta)=1, \\
\operatorname{sd}(\theta) & =0, \quad \operatorname{sd}(\theta)=1 .
\end{aligned}
$$

This grading is additive under composition and disjoint union. For any two generators $f$ and $g$ and the permutation Perm, we put

$f \otimes \mathrm{id}:=f \otimes \mathrm{id}, \quad \operatorname{id} \otimes f:=\operatorname{Perm} \circ(f \otimes \mathrm{id}) \circ$ Perm $, \quad f \otimes g:=(f \otimes \mathrm{id}) \circ(\mathrm{id} \otimes g)$

where $\otimes$ denotes the disjoint union. The composition rule is modified as follows:

$$
(\mathrm{id} \otimes g) \circ(f \otimes \mathrm{id})=(-1)^{\operatorname{sd}(\mathrm{f}) \operatorname{sd}(\mathrm{g})} f \otimes g .
$$

For an alternative description of OddCob, see Putyra's Master Thesis [13] using cobordisms with chronology.

Observe that the permutation does not commute with the monoidal product $\square$ in OddCob. Hence, when depicting a split as a chord attached to a circle, we need to fix an orientation on this chord, establishing the order of the two circles after the split. After this is done, the lists of commutative, anti-commutative and zero faces in OddCob and odd Khovanov homology coincide (after correcting the errors in Figure 2 [12]).

Let us denote by $\mathrm{Cob}_{0}$ the quotient of Cob by all morphisms of nonzero genus and spheres.

Lemma 4.2. OddCob is a $\mathbb{Z}_{2}$-extension of $\mathrm{Cob}_{0}$.

Proof. We have to show that the automorphism group of any 1-morphism is trivial. If the automorphism group of a certain 1-morphism was not trivial, it would contain -Id which would imply that under the EQFT of the next section the 1-morphism would be sent to zero. This is not the case for all 1-morphisms in $\mathrm{Cob}_{0}$.

4.2. Odd Frobenius system. In [12] an EQFT into the $\mathbb{Z} / 2 \mathbb{Z}$-graded abelian groups based on OddCob is constructed.

Using Khovanov's algebra $A_{0}=\mathbb{Z}[X] / X^{2}$, one can describe this EQFT $F_{0}$ : OddCob $\rightarrow \mathbb{Z}$-Mod as follows: $F_{0}$ maps a circle to $A_{0}$ where $A_{0}$ is $\mathbb{Z} / 2 \mathbb{Z}$ graded as follows: $\mathbb{1}$ is in degree 0 and $X$ is in degree 1 . To $n$ circles, $F_{0}$ assigns $A_{0}^{\otimes n}$. To generating morphisms, $F_{0}$ assigns the following maps:

$$
m:\left\{\begin{array}{l}
\mathbb{1} \otimes \mathbb{1} \mapsto \mathbb{1}, \\
\mathbb{1} \otimes X \mapsto X, \\
X \otimes \mathbb{1} \mapsto X, \\
X \otimes X \mapsto 0,
\end{array} \quad P:\left\{\begin{array}{l}
\mathbb{1} \otimes \mathbb{1} \mapsto \mathbb{1} \otimes \mathbb{1}, \\
\mathbb{1} \otimes X \mapsto X \otimes \mathbb{1}, \\
X \otimes \mathbb{1} \mapsto \mathbb{1} \otimes X, \\
X \otimes X \mapsto-X \otimes X,
\end{array} \quad \Delta:\left\{\begin{array}{l}
\mathbb{1} \mapsto X \otimes \mathbb{1}-\mathbb{1} \otimes X, \\
X \mapsto X \otimes X .
\end{array}\right.\right.\right.
$$


The maps $\varepsilon$ and $\eta$ are the same as in the Khovanov case.

Due to the fact that $\Delta$ and $\varepsilon$ have sd-degree $1, F_{0}$ cannot map the disjoint union of cobordisms to the tensor product of maps assigned to them since in this case relations (6) and (7) in Definition 4.1 would not be satisfied. Instead $F_{0}$ has to respect the monoidal product in OddCob and to map disjoint union to $\square$ defined by

$$
\begin{aligned}
f \otimes \mathrm{id} & :=f \otimes \mathrm{id}, \\
\mathrm{id} \otimes f & :=\text { Perm } \circ(f \otimes \mathrm{id}) \circ \text { Perm, } \\
f \otimes g & :=(f \otimes \mathrm{id}) \circ(\mathrm{id} \otimes g), \\
(\mathrm{id} \otimes g) \circ(f \otimes \mathrm{id}) & =(-1)^{\operatorname{deg}(f) \operatorname{deg}(g)} f \otimes g .
\end{aligned}
$$

The relations (6) and (7) hold now just by definition.

Applied to the Khovanov hypercube, this EQFT gives rise to a link homology theory, called odd Khovanov homology [12].

\section{References}

[1] M. M. Asaeda, J. H. Przytycki, and A. S. Sikora, Categorification of the Kauffman bracket skein module of I-bundles over surfaces. Algebr. Geom. Topol. 4 (2004), 1177-1210. MR 2113902 Zbl 1070.57008

[2] D. Bar-Natan, On Khovanov's categorification of the Jones polynomial. Algebr. Geom. Topol. 2 (2002), 337-370. MR 1917056 Zbl 0998.57016

[3] D. Bar-Natan, Khovanov's homology for tangles and cobordisms. Geom. Topol. 9 (2005), 1443-1499. MR 2174270 Zbl 1084.57011

[4] J. Carter and M. Saito, Frobenius module and essential surface cobordisms. Preprint 2009. arXiv:0905.4475 [math.GT]

[5] M. W. Hirsch, Differential topology. Graduate Texts in Math. 33, Springer-Verlag, New York 1994. MR 1336822 Zbl 0356.57001

[6] M. Khovanov, A categorification of the Jones polynomial. Duke Math. J. 101 (2000), 359-426. MR 1740682 Zbl 0960.57005

[7] M. Khovanov, Link homology and Frobenius extensions. Fund. Math. 190 (2006), 179-190. MR 2232858 Zbl 1101.57004

[8] J. Kock, Frobenius algebras and 2D topological quantum field theories. London Math. Soc. Stud. Texts 59, Cambridge University Press, Cambridge 2004. MR 2037238 Zbl 1046.57001

[9] A. Lauda, Frobenius algebras and planar open string topological field theories. Preprint 2005. arXiv:math/0508349 [math.QA]

[10] E. Lee, An endomorphism of the Khovanov invariant. Adv. Math. 197 (2005), 554-586. MR 2173845 Zbl 1080.57015

[11] S. Mac Lane, Categories for the working mathematician. 2nd ed., Graduate Texts in Math. 5, Springer-Verlag, New York 1998. MR 1712872 Zbl 0906.18001 
[12] P. Ozsváth, J. Rasmussen, and Z. Szabó, Odd Khovanov homology. Preprint 2007. arXiv:0710.4300 [math.QA]

[13] K. Putyra, Cobordisms with chronologies and a generalization of the Khovanov complex. Preprint 2010. arXiv:1004.0889 [math.AT]

[14] G. Schaefer, Master Thesis, University of Bonn, Bonn 2010.

[15] C. Schommer-Pries, The Classification of two-dimensional extended topological field theories. PhD thesis, UC Berkeley, Berkeley 2009.

http://sites.google.com/site/chrisschommerpriesmath/Home

[16] C. Stroppel, Convolution algebra and Khovanov homology. Talk at the Swiss Knots Conference, March 2009.

[17] C. Stroppel and B. Webster, 2-block Springer fibers: convolution algebras and coherent sheaves. Preprint 2008. arXiv:0802.1943 [math.RT]

[18] V. Turaev and P. Turner, Unoriented topological quantum field theory and link homology. Algebr. Geom. Topol. 6 (2006), 1069-1093. MR 2253441 Zbl 1134.57004

Received December 2, 2009; revised May 24, 2010

A. Beliakova, Institut für Mathematik, Universität Zürich, Winterthurerstrasse 190, 8057 Zürich, Switzerland

E-mail: anna@math.unizh.ch

E. Wagner, Institut de Mathématiques de Bourgogne, Université de Bourgogne, UMR 5584 du CNRS, BP 47870, 21078 Dijon Cedex, France

E-mail: emmanuel.wagner@u-bourgogne.fr 